\title{
GROUPS OF ELLIPTIC LINEAR FRACTIONAL TRANSFORMATIONS
}

\author{
R. C. LYNDON AND J. L. ULLMAN
}

1. Introduction. Our aim is to give a simple and self contained statement and proof of the following theorem:

Every subgroup of the group of linear fractional transformations of the complex plane, which contains only elliptic transformations, is conjugate to the image under stereographic projection of a group of rotations of a sphere.

An important consequence is that every discontinuous (or discrete) elliptic group is finite.

Simple proofs of these well-known results do not seem to be easily accessible. Indeed, we are indebted to J. Lehner for the only reference we know, to Fatou [1], where an argument similar to that given here is presented in a much fuller context.

2. Definitions and preliminaries. A linear fractional transformation is a map $T: z \rightarrow(a z+b) /(c z+d)$, of the (extended) complex plane onto itself, with complex coefficients and nonvanishing determinant. We may require $a d-b c=1$; then the matrix is determined up to a factor -1 , and we may write

$$
T= \pm\left(\begin{array}{ll}
a & b \\
c & d
\end{array}\right)
$$

These transformations form a group $\mathcal{L}$, with composition corresponding to matrix multiplication.

An elliptic transformation is one that is conjugate to a rotation $z \rightarrow k z,|k|=1$, of the complex plane. More explicitly, $T$ is elliptic if $T=I$, the identity map $z \rightarrow z$, or if it has distinct eigenvalues $\lambda$ and $\lambda^{-1}=\bar{\lambda}$ (complex conjugate) with $|\lambda|=1$. Then $T$ has trace $a+d$ $=\lambda+\bar{\lambda}$ real, with $|a+d| \leqq 2$. It follows also that $T$ has two distinct fixed points.

Consider the finite complex plane as a plane in three dimensional space with coordinate axes $(x, y, t)$ forming a right handed system. Let $\Sigma$ be the unit sphere, with equator the circle $|z|=1$ and north pole $N$. Stereographic projection carries each point $P \neq N$ on $\Sigma$ into the point $P^{\prime}$ where the line $N P$ meets the plane, with $N^{\prime}=\infty$. If $\widetilde{T}$ is a rotation of $\Sigma$; there is a unique transformation $T$ in $\&$ such that, when $\tilde{T}(P)=Q, T\left(P^{\prime}\right)=Q^{\prime}$. Let $R$ be the group of all linear fractional

Received by the editors August 18, 1966. 
transformations $T$ obtained in this way from a rotation $\tilde{T}$ of $\Sigma$.

Let $\tilde{A}_{\theta}$ denote the rotation of $\Sigma$ around the $t$ axis which turns the positive $x$ axis by an angle $\theta$ towards the positive $y$ axis. The corresponding element $A_{\theta}$ in $R$ is

$$
\left(\begin{array}{ll}
e^{i \theta / 2} & 0 \\
0 & e^{-i \theta / 2}
\end{array}\right) \text {. }
$$

Let $\widetilde{B}_{\phi}$ denote the rotation of $\Sigma$ around the $y$ axis which turns the positive $x$ axis by an angle $\phi$ towards the positive $t$ axis. The corresponding element $B_{\phi}$ in $R$ is

$$
\left(\begin{array}{cc}
\cos \frac{\phi}{2} & \sin \frac{\phi}{2} \\
-\sin \frac{\phi}{2} & \cos \frac{\phi}{2}
\end{array}\right)
$$

A general rotation $\widetilde{T}$ of $\Sigma$ will have a fixed point $P$ whose spherical coordinates are $(\phi, \theta)$, and a rotation angle $\alpha$. If $O$ is the origin, $\phi$ is the angle between $O N$ and $O P$ satisfying $0 \leqq \phi \leqq \pi$, while $\theta$ is the angle between the positive $x$ axis and the projection of $O P$ in the $x y$ plane measured counterclockwise and satisfying $0 \leqq \theta<2 \pi$. We take $0 \leqq \alpha$ $<2 \pi$ as the clockwise angle of rotation as viewed from the center of the sphere. Now $T$ can be written as the composition $(\widetilde{C})^{-1} \tilde{A}_{\alpha} \widetilde{C}$ where $\tilde{C}=\widetilde{B}_{-\phi} \tilde{A}_{-\theta}$ takes $P$ to $N$. The composition of the corresponding elements of $L$ yields

$$
\left(\begin{array}{cr}
\cos \frac{\alpha}{2}+i \sin \frac{\alpha}{2} \cos \phi & i \sin \frac{\alpha}{2} e^{i \theta} \sin \phi \\
i \sin \frac{\alpha}{2} e^{-i \theta} \sin \phi & \cos \frac{\alpha}{2}-i \sin \frac{\alpha}{2} \cos \phi
\end{array}\right) .
$$

We note that $T$ in $R$ has the form

$$
\left(\begin{array}{cc}
a & b \\
-\bar{b} & \bar{a}
\end{array}\right)
$$

with $|a|^{2}+|b|^{2}=1$. Conversely, if an element $T$ of $\mathscr{L}$ is of the form

$$
\left(\begin{array}{cc}
a & b \\
-\bar{b} & \bar{a}
\end{array}\right)
$$

with $|a|^{2}+|b|^{2}=1$, and is not the identity, then the equations

$$
\cos \frac{\alpha}{2}=\operatorname{Re} a \quad(0 \leqq \alpha<2 \pi)
$$




$$
\begin{aligned}
\theta & =\arg b-\frac{\pi}{2} \quad(0 \leqq \theta<2 \pi) \\
\sin \phi & =\frac{|b|}{\sqrt{ }\left(1-(\operatorname{Re} a)^{2}\right)}, \\
\cos \phi & =\frac{\operatorname{Im} a}{\sqrt{ }\left(1-(\operatorname{Re} a)^{2}\right)} \quad(0 \leqq \phi \leqq \pi)
\end{aligned}
$$

admit unique solutions in the indicated ranges. These numbers are the parameters of a rotation $\widetilde{T}$ whose corresponding element in $\mathscr{L}$ is $T$ and hence $T$ is in $R$.

We have shown that $T$ is in $R$ if and only if it has the form

$$
\left(\begin{array}{cc}
a & b \\
-\bar{b} & \bar{a}
\end{array}\right), \quad|a|^{2}+|b|^{2}=1
$$

by a method which yields at the same time the parameters of the corresponding rotation $\tilde{T}$.

3. The theorem. If $\mathcal{G}=\left(T_{0}, T_{1}, \cdots\right)$ is any subgroup of $\mathscr{L}$, and $U$ any element of $\mathcal{L}$, then $\mathcal{G}^{\prime}=\left(U T_{0} U^{-1}, U T_{1} U^{-1}, \cdots\right)$ is another subgroup, isomorphic to $\mathcal{G}$. (Indeed, we may think of $\mathcal{S}^{\prime}$ as obtained from $\mathcal{G}$ by the (conformal) change of coordinates $z \rightarrow u(z)$.) Any group $U \mathcal{G} U^{-1}$ is a conjugate of the group $\mathcal{G}$.

THEOREM. Let $\mathcal{G}$ be a subgroup of the linear fractional group $\mathfrak{L}$ such that $\mathcal{G}$ contains only elliptic transformations. Then some group $\mathcal{G}^{\prime}$ $=U \mathcal{G} U^{-1}$, conjugate to $\mathcal{G}$ by an element $U$ of $\mathcal{L}$, is contained in the $s u b$ group $\mathbb{R}$ of $\mathfrak{L}$, induced by rotations of the unit sphere.

4. The proof. Let there be given a group $\mathcal{G}$ of elliptic transformations. If $\mathcal{G}$ contains only the identity transformation

$$
I= \pm\left(\begin{array}{ll}
1 & 0 \\
0 & 1
\end{array}\right)
$$

the assertion becomes trivial. Assume that $\mathcal{G}$ contains some $T \neq I$. Then the quadratic equation $z=T(z)$ has distinct solutions $z_{1}$ and $z_{2}$, the fixed points of $T$. Now the conditions $U(0)=z_{1}$ and $U(\infty)=z_{2}$ have a solution $U$ in $\mathcal{L}$ (in fact we still have left one degree of freedom in the choice of $U$ ). Therefore the element $U^{-1} T U$ of $U^{-1} \mathcal{G} U$ has fixed points 0 and $\infty$. After replacing $\mathcal{G}$ by its conjugate $U^{-1} \mathcal{G} U$ we may assume that $\mathcal{G}$ contains some $T \neq I$ with fixed points 0 and $\infty$.

Let $\mathcal{G}_{0}$ be the subgroup of $\mathcal{G}$ consisting of all $T$ in $\mathcal{G}$ with 0 and $\infty$ as fixed points. The equations $T(0)=0$ and $T(\infty)=\infty$ imply $T$ is diagonal, 


$$
T=\left(\begin{array}{ll}
a & 0 \\
0 & d
\end{array}\right) .
$$

The condition $a d-b c=1$ gives $d=a^{-1}$. Since $T$ is elliptic, $|a|=1$, whence $d=\bar{a}$. Thus the elements of $\mathcal{G}_{0}$, of the form

$$
\left(\begin{array}{ll}
a & 0 \\
0 & \bar{a}
\end{array}\right)
$$

with $|a|=1$, belong to the group $R$ of all $T$ of the form

$$
T=\left(\begin{array}{cc}
a & b \\
-\bar{b} & \bar{a}
\end{array}\right) .
$$

We show next that every $T$ in $\mathcal{G}$ has the form

$$
T=\left(\begin{array}{ll}
a & b \\
c & \bar{a}
\end{array}\right) .
$$

Let

$$
T=\left(\begin{array}{ll}
a & b \\
c & d
\end{array}\right)
$$

and choose

$$
S=\left(\begin{array}{ll}
\lambda & 0 \\
0 & \bar{\lambda}
\end{array}\right)
$$

in $\mathcal{G}_{0}$, with $S \neq I$, hence with $\lambda \neq \pm 1$. Now

$$
S T=\left(\begin{array}{ll}
\lambda a & \lambda b \\
\bar{\lambda} c & \bar{\lambda} d
\end{array}\right)
$$

and the two elliptic elements $T$ and $S T$ of $g$ must have real traces, $a+d$ and $\lambda a+\bar{\lambda} d$. It follows first that $d=\bar{a}+h, h$ real, next that $\bar{\lambda} h$ is real, whence $h=0$ and $d=\bar{a}$.

We show that, for $T$ in $\mathcal{G}, b=0$ iff $c=0$. Suppose $c=0$, hence

$$
T=\left(\begin{array}{ll}
a & b \\
0 & \bar{a}
\end{array}\right)
$$

and take $S$ as before. Computation shows that, since $a \bar{a}=1$,

$$
S T S^{-1}=\left(\begin{array}{ll}
a & \lambda^{2} b \\
0 & \bar{a}
\end{array}\right) \quad \text { and } \quad T^{-1}=\left(\begin{array}{cc}
\bar{a} & -b \\
0 & a
\end{array}\right) .
$$

Then 


$$
K=S T S^{-1} T^{-1}=\left(\begin{array}{cc}
1 & \left(\lambda^{2}-1\right) b \\
0 & 1
\end{array}\right) .
$$

Since $K$, in $\mathcal{G}$, is elliptic and has eigenvalue 1 , we must have $K=I$, that is, $\left(\lambda^{2}-1\right) b=0$, and therefore $b=0$. The proof that $b=0$ implies $c=0$ is similar.

We now show that there exists a real number $r \neq 0$ such that every $T$ in $g$ has the form

$$
\left(\begin{array}{ll}
a & b \\
r \bar{b} & \bar{a}
\end{array}\right)
$$

For this it suffices to show that if $g$ contains elements

$$
T_{i}=\left(\begin{array}{cc}
a_{i} & b_{i} \\
r_{i} \bar{b}_{i} & \bar{a}_{i}
\end{array}\right), \quad i=1,2,
$$

with $b_{i} \neq 0, i=1,2$, then $r_{1}$ and $r_{2}$ are real and equal. Reality of $r_{i}$ follows from the condition that $a d-b c=a \bar{a}-r b \bar{b}=1$. We compute the diagonal elements of the product:

$$
T_{1} T_{2}=\left(\begin{array}{cc}
a_{1} a_{2}+r_{2} b_{1} \bar{b}_{2} & * \\
* & r_{1} \bar{b}_{1} b_{2}+\bar{a}_{1} \bar{a}_{2}
\end{array}\right) .
$$

These elements must be complex conjugates, whence $r_{2} b_{1} \bar{b}_{2}$ is conjugate to $r_{1} b_{1} b_{2}, r_{2} b_{1} b_{2}=r_{1} b_{1} \bar{b}_{2}$, and $r_{2}=r_{1}$.

We show that we may choose $r= \pm 1$. We have that every $T$ in $\mathcal{G}$ has the form

$$
\left(\begin{array}{ll}
a & b \\
r \bar{b} & \bar{a}
\end{array}\right)
$$

with real $r \neq 0$. Conjugating $\&$ by

$$
V=\left(\begin{array}{rr}
v & 0 \\
0 & v^{-1}
\end{array}\right)
$$

in $\mathcal{L}$, carries each $T$ into

$$
V T V^{-1}=\left(\begin{array}{ll}
a & v^{2} b \\
v^{-2} r \bar{b} & \bar{a}
\end{array}\right)
$$

with

$$
r^{\prime}=\left(v^{-2} r \bar{b}\right) /\left(\overline{\left.v^{2} b\right)}=r /(v \bar{v})^{2} .\right.
$$

Choosing $v$ such that $|v|^{4}=|r|$ gives $r^{\prime}= \pm 1$. (Here we have ex- 
ploited our remaining freedom in the choice of $U$.)

We show that $r=1$ is impossible unless each $T$ in $G$ has $b=c=0$. Suppose then that $r=+1$ and $\&$ contains

$$
T=\left(\begin{array}{ll}
a & b \\
b & \bar{a}
\end{array}\right)
$$

with $b \neq 0$. If

$$
S=\left(\begin{array}{ll}
\lambda & 0 \\
0 & \bar{\lambda}
\end{array}\right), \quad \lambda \neq \pm 1
$$

then

$$
\begin{aligned}
& S T S^{-1}=\left(\begin{array}{cc}
a & \lambda^{2} b \\
\bar{\lambda}^{2} \bar{b} & \bar{a}
\end{array}\right), \\
& T^{-1}=\left(\begin{array}{rr}
\bar{a} & -b \\
-\bar{b} & a
\end{array}\right),
\end{aligned}
$$

and

$$
K=\operatorname{STS}^{-1} T^{-1}=\left(\begin{array}{cc}
a \bar{a}-\lambda^{2} b \bar{b} & * \\
* & *
\end{array}\right) .
$$

Now the trace of $K$ is twice the real part of $a \bar{a}-\lambda^{2} b \bar{b}$, that is, twice $a \bar{a}+t b \bar{b}$, where $t$ is the real part of $-\lambda^{2}$, whence $a \bar{a}+t b \bar{b} \leqq 1$. But this is impossible, since $T$ has determinant $a \bar{a}-b \bar{b}=1$, while $-1<t<+1$.

We have shown that we may always take $r=-1$. Thus $G$ consists solely of transformations of the form

$$
T=\left(\begin{array}{rr}
a & b \\
-\bar{b} & \bar{a}
\end{array}\right),
$$

and $\mathcal{G}$ is a subgroup of $R$, as required.

\section{REFERENCE}

1. P. Fatou, Fonctions automorphes, Volume II of Théorie des fonctions algébriques, by P. Appel and E. Goursat, Gauthier-Villars, Paris, 1930.

UNIVERSITY OF MichigaN 